\title{
A transient boundary element method model of Schroeder diffuser scattering using well mouth impedance
}

\author{
Jonathan A. Hargreaves ${ }^{a)}$ and Trevor J. Cox \\ Acoustics Research Centre, The University of Salford, Manchester M5 4WT, United Kingdom
}

(Received 20 May 2008; revised 31 July 2008; accepted 19 August 2008)

\begin{abstract}
Room acoustic diffusers can be used to treat critical listening environments to improve sound quality. One popular class is Schroeder diffusers, which comprise wells of varying depth separated by thin fins. This paper concerns a new approach to enable the modeling of these complex surfaces in the time domain. Mostly, diffuser scattering is predicted using steady-state single frequency methods. A popular approach is to use a frequency domain boundary element method (BEM) model of a box containing the diffuser, where the mouth of each well is replaced by a compliant surface with appropriate surface impedance. The best way of representing compliant surfaces in time domain prediction models, such as the transient BEM is, however, currently unresolved. A representation based on surface impedance yields convolution kernels which involve future sound, so is not compatible with the current generation of time-marching transient BEM solvers. Consequently, this paper proposes the use of a surface reflection kernel for modeling well behavior and this is tested in a time domain BEM implementation. The new algorithm is verified on two surfaces including a Schroeder diffuser model and accurate results are obtained. It is hoped that this representation may be extended to arbitrary compliant locally reacting materials.
\end{abstract}

(C) 2008 Acoustical Society of America. [DOI: 10.1121/1.2982420]

PACS number(s): 43.55.Br, 43.20.Px, 43.20.Fn [SFW]

Pages: 2942-2951

\section{INTRODUCTION AND OVERVIEW}

Room acoustic diffusers can be used to treat critical listening environments to improve speech intelligibility and to make music sound better. ${ }^{1}$ Such devices are characterized by the uniformity of their scattering which may be measured under anechoic conditions, ${ }^{2}$ a time consuming and expensive process. An alternative is to predict this dispersion using a numerical model, and the boundary element method (BEM) is well suited to this task. ${ }^{3}$ The speed and low cost of this approach aid prototyping of new designs and even allow automated optimization of treatments to be performed. ${ }^{4}$ In a BEM model only the boundaries between obstacles and air are modeled as it is known how sound travels unobstructed. This produces smaller simpler meshes compared to volumetric methods such as finite element and finite difference time domain (FDTD). It also permits an unbounded volume of air to be modeled, making it ideal for free-field scattering scenarios.

Most BEMs assume harmonic excitation so the unknowns are time invariant and complex. While this frequency domain analyses is a useful tool, the transient behavior witnessed in the real world may only be recovered by solving many frequency domain models and then applying an inverse discrete Fourier transform (DFT). An alternative is to drop the time-invariant assumption and formulate the BEM in the time domain as is presented herein. This approach was first published by Friedman and Shaw in 1962, however, its implementation is problematic and consequently the method is still not in widespread use in acoustics. Some of the key issues are outlined below.

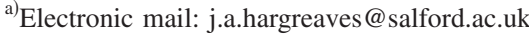

\section{A. Time domain BEM stability}

The discretized boundary integral equations (BIEs) forming the time domain BEM are typically solved by marching a solution on in time from known initial conditions, usually silence. However, being iterative this process has the potential for instability, a major impediment to the algorithm's widespread use. Rynne ${ }^{6}$ observed that similar instabilities affect all time domain BEM models regardless of the application, implying that this behavior is fundamental to the method rather than the problem considered.

The dominant analysis of this phenomenon is by the singularity expansion method. ${ }^{7}$ This expresses the system's response to excitation as a sum of resonant poles, each with its own natural frequency and damping. In discrete time, each pole is a complex scalar describing the magnitude and phase change the corresponding mode undergoes in a timestep; hence a mode with a pole of magnitude greater than unity will grow and cause the solver to diverge. These discrete poles are closely related to the eigenvalues of the statetransition process which may be found numerically. ${ }^{8-10}$ Such modes should be prohibited by the initial conditions, but in practice they can be seeded by numerical error in the solver; hence the onset of instability can appear highly random and implementation dependent. In addition, Rynne and Smith ${ }^{7}$ suggested that pole locations are perturbed by discretization error, so a pole of the BIE which is just stable may lead to a pole of the discretized system which is unstable.

When the problem of sound scattering from a body is stated as a BIE, the restriction that sound cannot travel through the body is lost and a continuation of the exterior medium, in this case, an air-filled cavity, is effectively created inside the body's bounding surface. At certain frequen- 
cies, this cavity may resonate, storing energy so the timeinvariant frequency domain BEM has a nonunique solution. In the time domain problem, these resonances correspond to oscillatory poles, borderline stable and likely candidates for corruption into divergence by numerical error. Such poles are not physically relevant so their removal is acceptable and improves solver performance. One method that achieves this in the frequency domain is the Burton and Miller formulation, ${ }^{11}$ and has been transferred to the time domain as the combined field integral equation (CFIE). ${ }^{12}$

The BEM is a wave based method and its computational cost increases rapidly with frequency. Acceleration algorithms ${ }^{13,14}$ have been published to address this issue but, as these are derived from the time-marching solvers for which instability issues remain, the focus herein remains on modeling smaller problems in a nonaccelerated fashion. In addition, some interesting work has been done on alternative solvers ${ }^{15-19}$ that may be less sensitive to divergent poles than the current time-marching generation.

\section{B. Modeling Schroeder diffusers}

The class of diffuser considered in this paper is the phase grating diffuser, whose development can be traced back to the pioneering work of Schroeder. ${ }^{20,21}$ These comprise a series of wells of differing depths according to a number theoretic sequence, separated by thin fins. Sound waves entering each well emerge following the time taken for them to travel to the bottom of the well, reflect, and travel back to the mouth. These delays are optimally decorrelated so the cumulative scattered sound is widely dispersed. Because the wells store sound energy and then reradiate it, the scattered sound is diffused in both space and time; recently, this transient behavior has begun to attract research interest. $^{22,23}$ In this paper, a one-dimensional diffuser based on the quadratic residue sequence will be considered; these are designed to diffuse in one plane only and take the form of an extruded cross section as depicted in Fig. 1(a), where the fins are shown partially transparent.

Modeling the two sides of a thin fin aggravates an issue in the BEM known as thin shape breakdown. ${ }^{24}$ This may be circumvented by using an open surface BEM which considers the surface to be comprised of thin rigid plates. This formulation has previously been implemented in the time domain ${ }^{25}$ however, it is unsuitable for modeling the solid part of the diffuser as it supports cavity resonances so is prone to instability.

Another modeling approach used in the frequency domain, which avoids these issues, is to model the mouth of each well as a compliant surface; thus the mesh is simplified and becomes a box enclosing the device [Fig. 1(b)]. A well's behavior is described by the surface impedance of its mouth, a quantity ideal for use with BEM, which may be found by assuming that all propagation in the well is axial with negligible losses, so every point at the well mouth reacts locally. At first, this may seem unrealistic, but it has been numerically shown to produce good results, ${ }^{26}$ and Schroeder's phase grating model makes these assumptions too. An equivalent time domain model is sought.
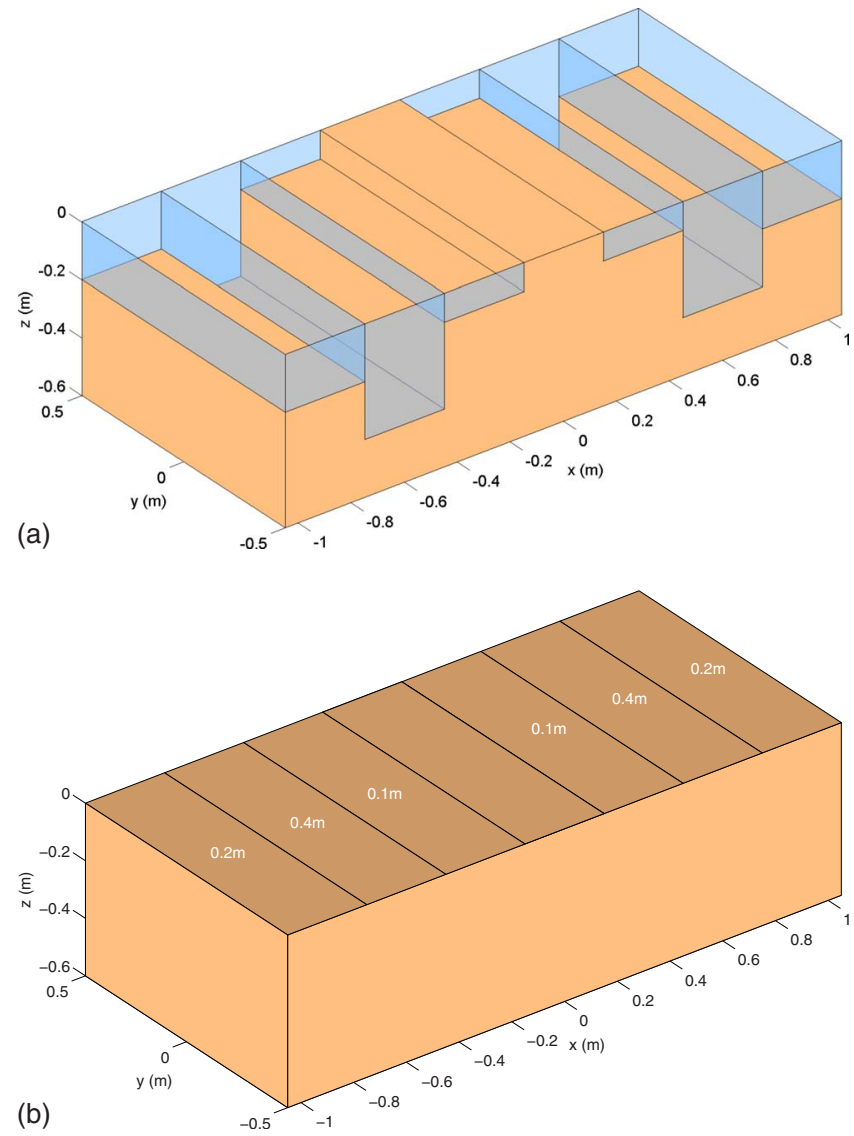

FIG. 1. (Color online) QRD (a) thin plate model, and (b) well mouth impedance model.

Differential boundary conditions may be used to model simple compliant materials such as broadband absorbers ${ }^{27,28}$ and limp membranes. ${ }^{29}$ However, finding such from arbitrary surface impedance data is more complicated, ${ }^{30}$ although Drumm and $\mathrm{Lam}^{31}$ successfully fitted infinite impulse response filters to surface absorption data. It has been suggested $^{32}$ that a convolution between waves traveling perpendicularly into and out of the body may be a more robust approach; this is adopted for the application herein and found to be effective.

This paper is structured as follows: Sec. II introduces the boundary integral formulation of the scattering problem and the CFIE. Section III introduces the new time domain well mouth model and describes its substitution into the BEM. The discretization process and time-marching solver are specified in Sec. IV. Verification results are shown and discussed in Sec. V followed by the conclusions in Sec. VI. Finally, details of the numerical integration procedure are outlined in the Appendix.

\section{BOUNDARY INTEGRAL EQUATION FORMULATIONS}

Figure 2 depicts a scattering problem, comprising an obstacle submerged in a connected medium $\Omega_{+}$with equilibrium density $\rho_{0}$ which obeys the linear acoustic wave equation with speed of sound $c . S$ is a surface conformal to the obstacle and sufficiently close that the obstacle's surface properties may be ascribed to it; thus the obstacle resides in the interior domain $\Omega_{-} . S_{\infty}$ is the extent of the medium. $\mathbf{x}$ and 


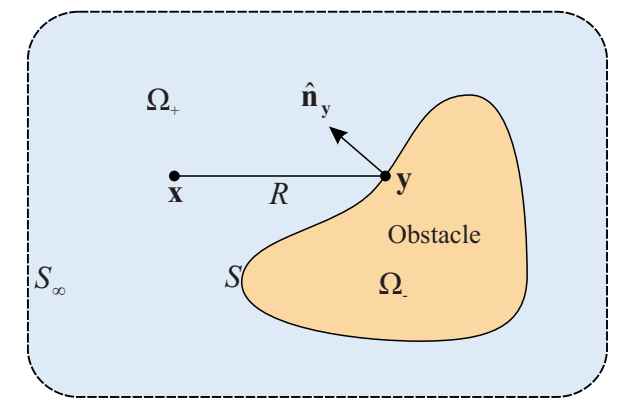

FIG. 2. (Color online) A scattering problem comprising an obstacle submerged in a connected medium. $S$ is a surface conformal to the obstacle; hence the medium is said to be external to $S$.

$\mathbf{y}$ are the three-dimensional Cartesian vectors defining the observation and radiation points respectively, $R=|\mathbf{x}-\mathbf{y}|$ is the distance between them, and $\hat{\mathbf{n}}_{\mathbf{y}}$ is the surface normal unit vector at $\mathbf{y}$.

Sound is represented by the velocity potential $\varphi$ which, while not a physical quantity, has the convenient property that both pressure $p$ and particle velocity $\mathbf{v}$ may be derived from it:

$$
\begin{aligned}
& p(\mathbf{x}, t)=-\rho_{0} \dot{\varphi}(\mathbf{x}, t), \\
& \mathbf{v}(\mathbf{x}, t)=\nabla \varphi(\mathbf{x}, t),
\end{aligned}
$$

where $t$ is the time and a dot above a quantity indicates temporal differentiation. An incident disturbance $\varphi_{i}(\mathbf{x}, t)$ exists in $\Omega_{+}$but does not reach the obstacle while $t \leqslant 0$. When $\varphi_{i}(\mathbf{x}, t)$ does reach the obstacle, a wave $\varphi_{s}(\mathbf{x}, t)$ is scattered such that the total disturbance $\varphi_{t}(\mathbf{x}, t)=\varphi_{i}(\mathbf{x}, t)+\varphi_{s}(\mathbf{x}, t)$ matches the surface properties of the obstacle; thus this is an initial-boundary-value problem. Application of Green's theorem $^{33}$ allows the propagation of $\varphi_{s}(\mathbf{x}, t)$ in $\Omega_{+}$to be stated as the Kirchhoff integral equation (KIE) over its boundary $S \cup S_{\infty}$. In practice, $S_{\infty}$ is chosen so distant that its contribution does not arrive within the modeling duration, so the integration domain may be reduced to $S$. This statement describes scattered velocity potential in a manner equivalent to the classical Huygens principle, being the propagation of the wavefront at each point on the boundary summed together:

$$
\begin{aligned}
\varphi_{S}(\mathbf{x}, t)= & \iint_{S}\left[\varphi_{t}(\mathbf{y}, t)^{*} \hat{\mathbf{n}}_{\mathbf{y}} \cdot \nabla_{\mathbf{y}} g(R, t)\right. \\
& \left.-g(R, t)^{*} \hat{\mathbf{n}}_{\mathbf{y}} \cdot \nabla_{\mathbf{y}} \varphi_{t}(\mathbf{y}, t) d \mathbf{y}\right] .
\end{aligned}
$$

The term $\hat{\mathbf{n}}_{\mathbf{y}} \cdot \nabla_{\mathbf{y}} \varphi_{t}(\mathbf{y}, t)$ is the surface normal component of the particle velocity and is termed "normal velocity" for brevity. Despite the relation given in Eq. (2), normal velocity and velocity potential are independent fields on $S$ since surface normal derivatives cannot be found from quantities only known on a surface. $*$ denotes temporal convolution and $g(R, t)$ is the time domain Green's function, which describes how sound travels from an instantaneous point source at $t$ $=0$ to a point observer at distance $R$, given by

$$
g(R, t)=\frac{\delta(t-R / c)}{4 \pi R},
$$

where $\delta(\cdots)$ is a Dirac delta function. This delay term in the numerator encapsulates the finite propagation speed of sound, thereby dictating the domain of dependence of the scattered wave in Eq. (3) and ensuring causality in the resulting algorithm.

Consideration of the obstacle's boundary condition at $\mathbf{x}$ allows solution for the total surface sound, from which scattered sound at any desired off-surface point may be evaluated.

Specifically the CFIE is equivalent to the boundary condition $(1-\alpha) p_{t}(\mathbf{x}, t)=\alpha \rho_{0} c \hat{\mathbf{n}}_{\mathbf{x}} \cdot \mathbf{v}_{t}(\mathbf{x}, t)$ when the limit is taken as $\mathbf{x}$ approaches $S$ from the inside. The corresponding integral operator may be expressed as

$$
\begin{aligned}
L_{c}\left\{\varphi_{t}(\mathbf{x}, t)\right\}= & (1-\alpha) \dot{\varphi}_{i}(\mathbf{x}, t)+\alpha c \hat{\mathbf{n}}_{\mathbf{x}} \cdot \nabla \varphi_{i}(\mathbf{x}, t) \\
= & -\left[(1-\alpha) \frac{\partial}{\partial t}+\alpha c \hat{\mathbf{n}}_{\mathbf{x}} \cdot \nabla_{\mathbf{x}}\right] \\
& \times \iint_{S}\left[\varphi_{t}(\mathbf{y}, t)^{*} \hat{\mathbf{n}}_{\mathbf{y}} \cdot \nabla_{\mathbf{y}} g(R, t)\right. \\
& \left.-g(R, t)^{*} \hat{\mathbf{n}}_{\mathbf{y}} \cdot \nabla_{\mathbf{y}} \varphi_{t}(\mathbf{y}, t)\right] d \mathbf{y} \quad \text { if } \mathbf{x} \in S_{-} .
\end{aligned}
$$

This formulation is stated ${ }^{12}$ to be the time domain equivalent to the Burton and Miller formulation ${ }^{11}$ commonly used in frequency domain acoustic BEMs. It differs slightly from the latter, in particular, with regard to the range of values taken by the real scalar blend parameter $\alpha$, and matches more closely its namesake in the electromagnetic BEM formulation. ${ }^{34}$

When $\alpha=\frac{1}{2}$ the boundary condition which founds the CFIE simplifies to $p_{t}(\mathbf{x}, t) / \hat{\mathbf{n}}_{\mathbf{x}} \cdot \mathbf{v}_{t}(\mathbf{x}, t)=\rho_{0} c$, a condition satisfied by any plane wave propagating in the direction of $\hat{\mathbf{n}}_{\mathbf{x}}$, that is out of the cavity. More generally, it has been shown that when $0<\alpha<1$ energy flows out of the cavity and it cannot support resonant modes. ${ }^{35}$ Consequentially, $L_{c}$ has been shown to grant stability superior to alternate operators for a variety of test geometries. Therefore, it is desirable to derive the BEM algorithm from the CFIE for all compatible scattering obstacles.

\section{WELL MOUTH SURFACE MODEL}

Consider a well of constant cross section and depth $d$, as depicted in Fig. 3. It is assumed that the width of the well is small with respect to wavelength so that only axial plane wave propagation is supported. Accordingly, the sound in the well may be expressed as the superposition of two plane waves traveling into and out of the well, designated $\varphi_{\text {in }}$ and $\varphi_{\text {out }}$. The rigid boundary condition at the base of the well dictates that the incoming wave is reflected, such that the outgoing wave is the incoming wave with a fixed time delay.

Under the assumption of time-harmonic oscillation $\varphi(z, t)=\operatorname{Re}\left\{e^{-i \omega t} \Phi(z, \omega)\right\}$, the mouth of the well may be characterized by this well known expression for surface impedance at the well mouth $(z=0)$ : 


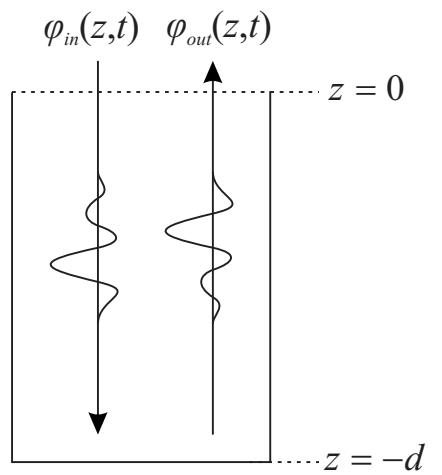

FIG. 3. A simple well with constant cross section, depth $d$, and mouth at $z=0$.

$$
Z(\omega)=\frac{P_{t}(0, \omega)}{V_{t, \text { in }}(0, \omega)}=i \rho_{0} c \cot (k d),
$$

where $k=\omega c^{-1}$ is the wavenumber, $P_{t}(z, \omega)$ is the total pressure, and $V_{t, \text { in }}(z, \omega)$ is the inward component of total particle velocity. It is an ideal means of characterizing materials within a frequency domain BEM as it relates the two surface unknowns, pressure and normal velocity, by a complex scalar allowing the problem to be simplified to having only one surface unknown.

The same relationship may be stated in the time domain as $p_{t}(0, t)=v_{t, \text { in }}(0, t)^{*} z(t)$. However, a $z(t)$ found by inverse DFT of $Z(\omega)$ is typically noncompact in time and requires future values of $v_{t, \text { in }}(0, t)$. This is due to the aggregation of cause and effect in the quantities $p_{t}(0, t)$ and $v_{t, \text { in }}(0, t)$, and means that this form cannot be used with a time-marching solver. Further to this, the inverse Fourier transform of Eq. (6) appears to be a nontrivial operation.

Another approach is to relate the incoming and outgoing waves at the mouth of the well as $\Phi_{\text {out }}(0, \omega)$ $=\Phi_{\text {in }}(0, \omega) W(\omega)$, where $W(\omega)=e^{i 2 k d}$ is the surface reflection coefficient. This too may be stated in the time domain:

$$
\varphi_{\text {out }}(0, t)=\varphi_{\text {in }}(0, t)^{*} w(t)
$$

where the time-invariant surface reflection kernel $w(t)$ is typically compact in time and expresses $\varphi_{\text {out }}$ using only past values of $\varphi_{\text {in }}$, hence is suitable for use with a time-marching solver. For the well model above,

$$
w(t)=\delta\left(t-2 d c^{-1}\right)
$$

Equations (7) and (8) will be used as the basis for the development of a time domain well mouth surface model.

\section{A. Surface reflection boundary condition}

Surface impedance is considered to vary spatially so $\hat{\mathbf{n}}_{\mathbf{x}} \cdot \nabla \Phi_{t}(\mathbf{x}, \omega)=-i \omega \rho_{0} \Phi_{t}(\mathbf{x}, \omega) Z(\mathbf{x}, \omega)^{-1}$ holds for every point on $S$. Implicit in this is the assumption that the obstacle reacts locally. An equivalent time domain boundary condition may be written using the surface reflection kernel form of Eq. (7):

$$
\varphi_{\text {out }}(\mathbf{x}, t)=\varphi_{\text {in }}(\mathbf{x}, t)^{*} w(\mathbf{x}, t) .
$$

The total velocity potential used in Sec. II is the sum of the incoming and outgoing waves:

$$
\varphi_{t}(\mathbf{x}, t)=\varphi_{\text {in }}(\mathbf{x}, t)+\varphi_{\text {out }}(\mathbf{x}, t)=\varphi_{\text {in }}(\mathbf{x}, t)^{*}[\delta(t)+w(\mathbf{x}, t)] .
$$

This form suggests that $\varphi_{\text {in }}(\mathbf{x}, t)$ should be discretized as it is the fundamental surface unknown and $\varphi_{\text {out }}(\mathbf{x}, t)$ and $\varphi_{t}(\mathbf{x}, t)$ are the secondary effects of its impingement on the obstacle. Similar statements may be found for total pressure and total normal velocity:

$$
p_{t}(\mathbf{x}, t)=-\rho_{0} \dot{\varphi}_{t}(\mathbf{x}, t)=-\rho_{0} \frac{\partial}{\partial t}\left[\varphi_{\text {in }}(\mathbf{x}, t)^{*}[\delta(t)+w(\mathbf{x}, t)]\right],
$$

$$
\begin{aligned}
\hat{\mathbf{n}}_{\mathbf{x}} \cdot \nabla \varphi_{t}(\mathbf{x}, t) & =\hat{\mathbf{n}}_{\mathbf{x}} \cdot \nabla\left[\varphi_{\text {in }}(\mathbf{x}, t)+\varphi_{\text {out }}(\mathbf{x}, t)\right] \\
& =\left.\frac{\partial}{\partial z}\left[\varphi_{\text {in }}\left(\mathbf{x}, t+z c^{-1}\right)+\varphi_{\text {out }}\left(\mathbf{x}, t-z c^{-1}\right)\right]\right|_{z=0} \\
& =\frac{1}{c} \frac{\partial}{\partial t}\left[\varphi_{\text {in }}(\mathbf{x}, t)^{*}[\delta(t)-w(\mathbf{x}, t)]\right] .
\end{aligned}
$$

The above statements have been written without specifying $w(\mathbf{x}, t)$ in anticipation that they may be capable of describing a broader class of scattering obstacle. For the welled rigid surface model discussed $w(\mathbf{x}, t)=\delta\left(t-2 c^{-1} d(\mathbf{x})\right)$, where $d(\mathbf{x})=0$ for rigid nonwell surfaces sections. The sifting property of the delta function is exploited to simplify the above:

$$
\begin{aligned}
& \varphi_{t}(\mathbf{x}, t)=\varphi_{\text {in }}(\mathbf{x}, t)+\varphi_{\text {in }}\left(\mathbf{x}, t-2 c^{-1} d(\mathbf{x})\right), \\
& p_{t}(\mathbf{x}, t)=-\rho_{0}\left[\dot{\varphi}_{\text {in }}(\mathbf{x}, t)+\dot{\varphi}_{\text {in }}\left(\mathbf{x}, t-2 c^{-1} d(\mathbf{x})\right)\right], \\
& \hat{\mathbf{n}}_{\mathbf{x}} \cdot \nabla \varphi_{t}(\mathbf{x}, t)=\frac{1}{c}\left[\dot{\varphi}_{\text {in }}(\mathbf{x}, t)-\dot{\varphi}_{\text {in }}\left(\mathbf{x}, t-2 c^{-1} d(\mathbf{x})\right)\right] .
\end{aligned}
$$

These statements do not enforce that quantities are invariant over the cross section of the well as should be the case for plane waves; that restriction is left to the discretization scheme. Instead they state that the waves in the well are one dimensional, and that each point on the mouth of the well reacts locally. Radiation impedance of the well is accounted for in the boundary integral description of the problem. Substitution into Eq. (5) creates an operator that can calculate the sound scattered by an obstacle comprising rigid and welled sections while not supporting cavity resonances:

$$
\begin{aligned}
& (1-\alpha) \dot{\varphi}_{i}(\mathbf{x}, t)+\alpha c \hat{\mathbf{n}}_{\mathbf{x}} \cdot \nabla \varphi_{i}(\mathbf{x}, t) \\
& \quad=L_{c}\left\{\varphi_{\text {in }}(\mathbf{x}, t)+\varphi_{\text {in }}\left(\mathbf{x}, t-2 c^{-1} d(\mathbf{x})\right)\right\} \quad \text { if } \mathbf{x} \in S_{-} .
\end{aligned}
$$

In the following section, this new operator will be discretized to form a time-marching BEM.

\section{THE MARCHING-ON-IN-TIME METHOD}

The surface quantities must be discretized in order for a solution to the boundary conditions on $S$ to be found numerically. As suggested by Eq. (10), to ensure compatibility with 
the new surface reflection boundary condition, $\varphi_{\text {in }}(\mathbf{x}, t)$ will be discretized in preference to the usual $\varphi_{t}(\mathbf{x}, t)$. Otherwise the discretization scheme follows that used in Ref. 12.

The surface $S$ is partitioned into $N_{s}$ flat elements denoted $S_{n}$, all small with respect to the anticipated spatial variation of the sound field, and time is discretized into $N_{t}$ regular time-steps with duration $\Delta_{t}$. Discretization of the incoming wave is achieved by approximating it by a weighted summation of basis functions:

$$
\varphi_{\text {in }}(\mathbf{x}, t)=\sum_{n=1}^{N_{s}} \sum_{i=1}^{N_{t}} w_{n, i} f_{n}(\mathbf{x}) T_{i}(t)
$$

where $w_{n, i}$ are the discretization weights,

$$
f_{n}(\mathbf{x})= \begin{cases}1 & \text { if } \mathbf{x} \in S_{n} \\ 0 & \text { otherwise }\end{cases}
$$

are the spatial basis functions, and

$$
T_{i}(t)=T\left(t-i \Delta_{t}\right)
$$

are the temporal basis functions, the latter being regularly delayed copies of the mother basis function $T(t)$. Currently $T(t)$ is chosen to be the piecewise polynomial used in Ref. 12.

This discretization scheme is substituted into Eq. (16) and the summations and weights are brought outside $L_{c}$. Collocation is performed in space and time to form a matrix equation; evaluation at $\mathbf{x}_{m}$ (the center of element $S_{m}$ ) and $t_{j}$ $=j \Delta_{t}$ contributes a row to

$$
\mathbf{Z}_{0} \mathbf{w}_{j}=\mathbf{e}_{j}-\sum_{l=1}^{\infty} \mathbf{Z}_{l} \mathbf{w}_{j-l},
$$

where $l=j-i$ is the retardation index and the weights $\mathbf{w}_{i ; n}$ $=w_{n, i}$. The interaction matrices are defined as follows:

$$
\mathbf{Z}_{l ; m, n}=L_{c}\left\{f_{n}(\mathbf{x})\left\lfloor T_{j-l}\left(t_{j}\right)+T_{j-l}\left(t_{j}-2 c^{-1} d_{n}\right)\right\rfloor\right\}, \quad \mathbf{x}=\mathbf{x}_{m-},
$$

where $d_{n}$ is the well depth of element $S_{n}$ and equals zero for rigid nonwell elements. These are evaluated efficiently and accurately by regularization to contour integrals and adaptive numerical integration; details are included in the Appendix. The excitation vectors are evaluated as

$$
\mathbf{e}_{j ; m}=(1-\alpha) \dot{\varphi}_{i}\left(\mathbf{x}_{m}, t_{j}\right)+\alpha c \hat{\mathbf{n}}_{\mathbf{x}} \cdot \nabla \varphi_{i}\left(\mathbf{x}_{m}, t_{j}\right) .
$$

This algorithm is commonly referred to as the marching on in time (MOT) or "retarded potential" algorithm and intuitively possesses an iterative structure with sound traveling from element to element with a finite speed. It may more generally be considered to be a matrix solver between excitation coefficients and discretization weights, which exploits a pattern in the interaction matrices due to the regular temporal basis functions.

Discretization accuracy may be quantified spatially and temporally by considering the maximum frequency $\omega_{\max }$ present in the incident wave. The maximum phase variation over an element with largest dimension $\Delta_{x}$ in a time-step is $\omega_{\max }\left(\Delta_{t}+\Delta_{x} c^{-1}\right)$. The logical assumption that spatial and temporal discretization error should be of similar magnitudes suggests the choice $\Delta_{x} \approx c \Delta_{t}$, as favored by Bluck and Walker. ${ }^{8}$ This leads to nonzero off diagonals in the matrix $\mathbf{Z}_{0}$, necessitating a matrix solution at each time step. $\mathbf{Z}_{0}$ will in practice be very sparse and an iterative matrix solver seeded with the previous time-step's weights provides an efficient implementation.

In the following section, the new time domain well mouth surface model will be verified against frequency domain BEM implementations.

\section{RESULTS}

The new algorithm implements a time domain BEM model of a welled obstacle characterized by the new surface reflection boundary condition. This has required development of the integration scheme but the numerical machinery is otherwise as previously published. Verifying this algorithm's results will demonstrate that the new surface reflection boundary condition is performing correctly.

Verification is achieved by comparison with frequency domain BEM implementations which have previously been shown to accurately match experimental data. ${ }^{36}$ Two scattering problems are considered, both of which involve obstacles possessing wells, the first being an object with uniform depth wells covering one face and the second being a Schroeder diffuser. A harmonic point source illuminates the surface for sufficient duration that the system reaches steady state and any instability has the opportunity to appear. The DFT is applied to the time domain data and the error versus the frequency domain BEM is quantified at the frequency of excitation. Using single frequency excitation is clearly an uninspiring application of the time domain BEM but is being done purely to achieve rigorous verification.

Two frequency domain BEM implementations are used. The closed body version models the well mouths as surface impedances according to Eq. (6). The open body version is capable of modeling the thin fins separating the wells so that the wells are modeled explicitly.

One mesh is used for each surface, and the time domain BEM is verified for a wide range of time-step durations defined by their relationship to spatial resolution, denoted implicitness $c \Delta_{t} \Delta_{x}^{-1}$. This is done because time-step duration has been associated with stability in many publications; evidently different values affect whether poles are perturbed into divergence. For each of these, a far-field harmonic point source, located $100 \mathrm{~m}$ distant normal to the obstacle, excites the system at a frequency such that the number of time-steps per excitation period $\beta=2 \pi\left(\Delta_{t} \omega\right)^{-1}$ assumes a range of predetermined values. For each combination, the error $e$ between the time and frequency domain BEMs is calculated from the normalized mean complex difference between the respective source-to-collocation-point transfer functions at the excitation frequency:

$$
e(\omega)=\frac{\sum_{m=1}^{N_{s}}\left|H_{\mathrm{TD}}\left(\mathbf{x}_{m}, \omega\right)-H_{\mathrm{FD}}\left(\mathbf{x}_{m}, \omega\right)\right|}{\sum_{m=1}^{N_{s}}\left|H_{\mathrm{FD}}\left(\mathbf{x}_{m}, \omega\right)\right|} .
$$

In the frequency domain, the transfer function $H_{\mathrm{FD}}$ is simply the total pressure divided by the source monopole pressure: 


$$
H_{\mathrm{FD}}(\mathbf{x}, \omega)=\frac{P_{t}(\mathbf{x}, \omega)}{P_{\text {source }}}
$$

In the time domain $H_{\mathrm{TD}}$ is found by division of the DFT of the total velocity potential by the DFT of the source monopole potential:

$$
H_{\mathrm{TD}}(\mathbf{x}, \omega)=\frac{F\left\{\varphi_{t}(\mathbf{x}, t)\right\}(\omega)}{F\left\{\varphi_{\text {source }}(t)\right\}(\omega)}
$$

The first $50 \beta$ (defined below) iterations are omitted from the DFT to allow the time domain solution to reach steady state. The next $100 \beta$ iterations are chosen for DFT; this length maintains periodicity and eliminates windowing error. This error ratio is displayed for each integration type as a percentage contour plot between time-step implicitness and temporal resolution $\beta$.

\section{A. Uniform welled body}

This body is a box $1.0 \mathrm{~m}^{2}$ by $0.5 \mathrm{~m}$ deep and its front face is covered by a lattice of wells all $0.1 \mathrm{~m}$ deep. Figure 4(a) shows the open surface mesh comprising 580 thin plate elements, and Fig. 4(b) shows the equivalent surface impedance mesh comprising 400 elements; the thin elements are partially transparent and the well mouth elements are colored darker. $\Delta_{x}=0.1 \mathrm{~m}$ for both meshes. The surface impedance model requires roughly half the memory and computation time required by the thin plate model.

Figure 5 shows the error between the time domain and frequency domain models of the mesh in Fig. 4(b) versus time-step implicitness and temporal resolution. To the left of the figure where the time-step duration is explicit, spatial resolution is poor with respect to excitation wavelength so the accuracy of all BEM suffers. Toward the bottom of the figure, temporal resolution of the excitation frequency is poor; error here primarily originates from the time domain BEM. However, in the middle to upper right quadrant of the figure, discretization error is low and good agreement occurs. No instability is witnessed for any time-step duration indicating that the CFIE operator has successfully avoided supporting any cavity resonances even when combined with the new well mouth surface model.

Figure 6 shows the interference patterns that occur between incident and scattered sound as further evidence that the well mouth surface model is behaving as expected. The receivers are arranged in a vertical line that starts behind the obstacle (left of the figure), passes through its center, and emerges at the front (right of the figure). They are spaced such that none touches the surface. The magnitude of the source to receiver transfer function $H$ is plotted in decibel versus the receiver $z$ coordinate. Data series are shown for the surface impedance mesh [Fig. 4(b)] modeled by the time domain BEM (TD impedance) and the frequency domain BEM for closed surfaces (FD impedance), and for the thin plate mesh [Fig. 4(a)] modeled by the frequency domain BEM for open surfaces (FD thin plate). The vertical lines at $z=0.0$ and $z=-0.5$ indicate the front and back of the obstacle and the shaded area indicates the wells of the mixed mesh. Time-step duration was chosen such that $\Delta_{x}=c \Delta_{t}$.
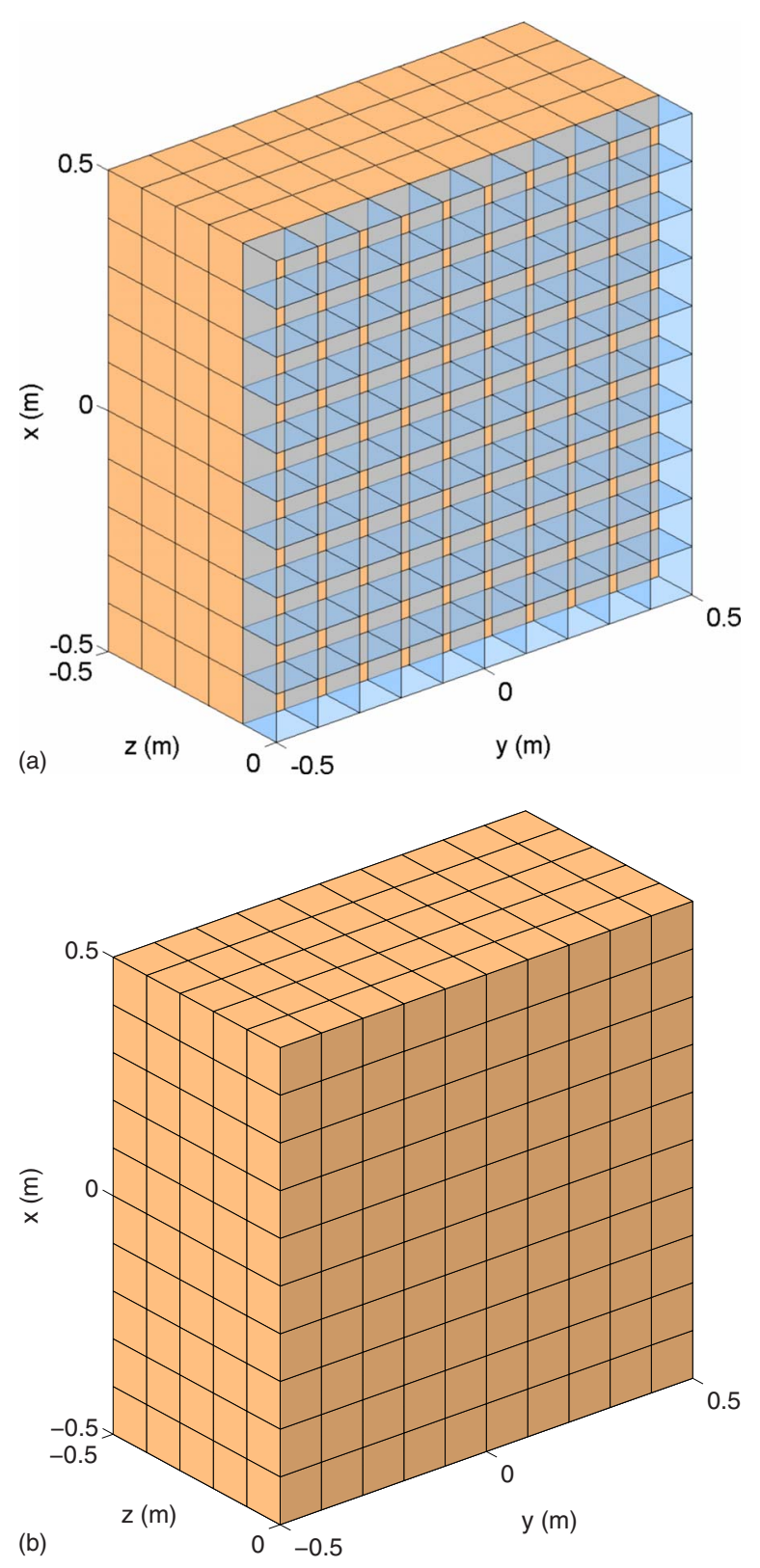

FIG. 4. (Color online) Meshes of a body with uniform depth wells on its front face: (a) thin plate model and (b) surface impedance model.

Interference effects between the incident and scattered waves are evident in front of the surface and in this region there is excellent agreement between the time and frequency domain algorithms. The BEM for open surfaces is seen to extend the interference patterns into the welled region and its surface normal gradient approaches zero as expected from the rigid well floors. Inside the surface, the frequency domain BEM for closed surfaces achieves the best cancellation, but the results still confirm that the time domain surface reflection response boundary condition does not permit sound to flow into the cavity. In the shadow region behind the obstacle, all models, roughly agree but there is no apparent interference behavior.

This modeling problem has shown excellent stability and agreement with a verified frequency domain BEM on a simple surface with welled sections. A more complex realworld device will now be modeled. 


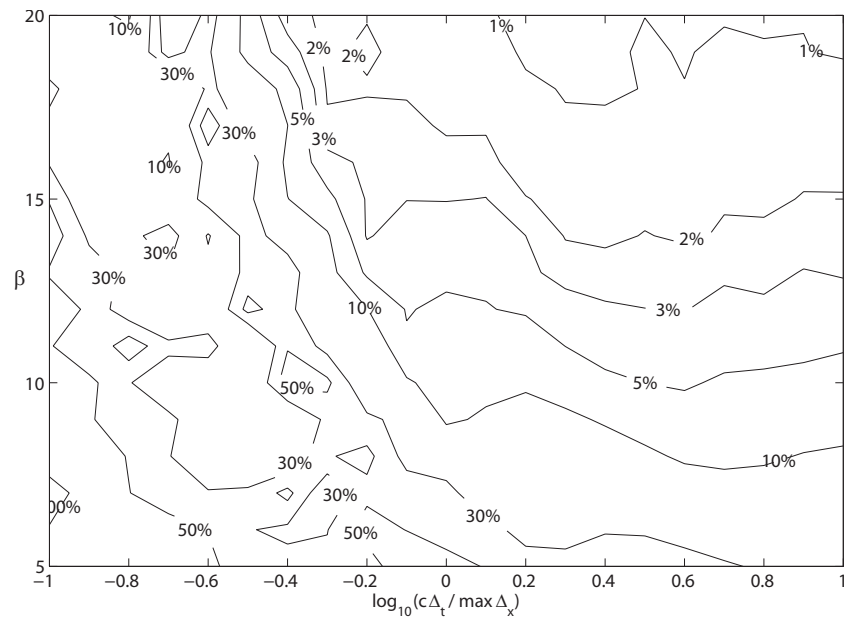

FIG. 5. Error between time and frequency domain BEMs for closed surfaces both modeling the uniform welled surface.

\section{B. Quadratic residue diffuser}

This device modeled has a design wavelength of $1.4 \mathrm{~m}$, a well width of $0.3 \mathrm{~m}$, a height of $1.0 \mathrm{~m}$ and follows the quadratic residue depth sequence $\left[\begin{array}{lllllll}2 & 4 & 1 & 0 & 1 & 4 & 2\end{array}\right]$. A diagram of the real device and the equivalent surface impedance model were shown in Figs. 1(a) and 1(b), respectively. $\Delta_{x}$ $=0.1 \mathrm{~m}$ for both meshes giving them 972 and 792 elements, respectively; consequentially, the surface impedance model requires roughly two-thirds of the memory and computation time of the thin plate model.

The relationship between the quadratic residue diffuser (QRD) meshes does not exactly mirror that between the uniform welled body meshes. Specifically, the well mouths of the surface impedance model mesh have been discretized into elements with the same spatial resolution as the rigid parts of the surface; this is a standard technique used to mesh compliant surfaces in the frequency domain. Each element reacts locally which is equivalent to having the wells partitioned by a lattice structure similar to that present on the front face of Fig. 4. This is not quite equivalent to either the

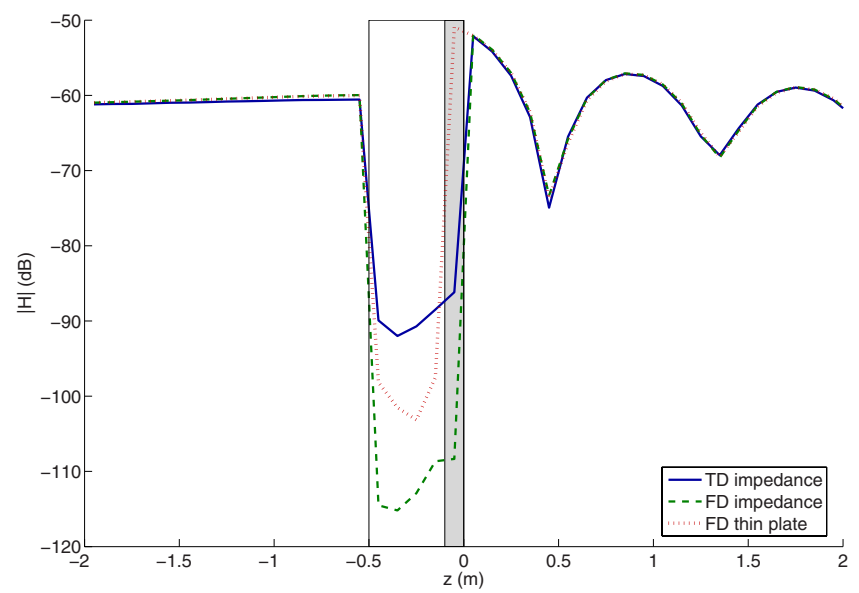

FIG. 6. (Color online) Total receiver sound though the uniform welled surface at $202 \mathrm{~Hz} \equiv \beta=12$. The vertical lines indicate the front and back of the obstacle and shading the wells on its front face.

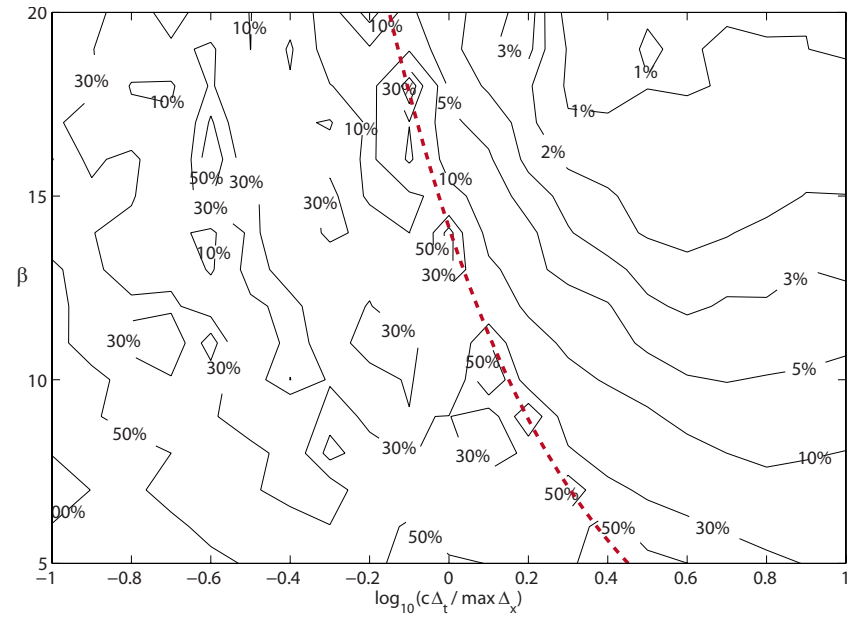

FIG. 7. (Color online) Error between the time and frequency domain BEM for closed surfaces on the surface impedance model of the QRD. The dashed line indicates the first modal frequency along the wells of the QRD.

real device, the model in Fig. 1(a), or Schroeder's plane wave model, but it has been shown to produce reasonable results. ${ }^{3}$

Figure 7 shows the error between the time and frequency domain implementations of the surface impedance model of the QRD depicted in Fig. 1(b). It is plotted versus time-step implicitness and temporal resolution. The trends are the same as observed for the uniform welled surface, with universal stability and good agreement in the upper right quadrant, indicating that the new algorithm is functioning correctly. The first modal frequency along the wells of the QRD $(171.5 \mathrm{~Hz}, \lambda=2 \mathrm{~m})$ is indicated by a dashed line and increased error can be observed close to it. This was an unexpected result since the well mouth impedance model effectively partitions the well, and its significance is an ongoing research question.

Figure 8 shows the magnitude of the source to receiver scattered sound transfer function in decibel versus receiver angle relative to the surface normal. This is calculated according to Eqs. (24) and (25) with the modification that scattered pressure and velocity potential are used in place of their total sound counterparts. The 91 receivers are uniformly spaced in a $5 \mathrm{~m}$ radius arc located in the primary scattering plane of the QRD. Data series are shown for the surface impedance mesh [Fig. 1(b)] modeled by the time domain

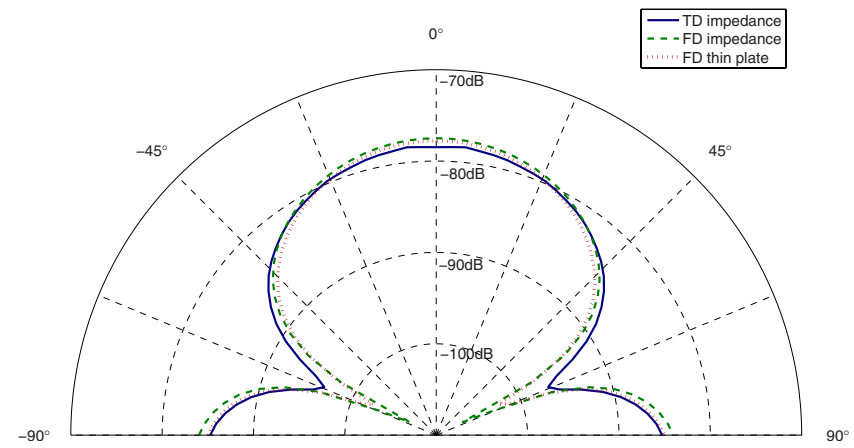

FIG. 8. (Color online) Scattered sound $(\mathrm{dB})$ at a receiver arc $5 \mathrm{~m}$ from the $\mathrm{QRD}$ at $202 \mathrm{~Hz} \equiv \beta=12$. 
BEM (TD impedance) and the frequency domain BEM for closed surfaces (FD impedance), and for the thin plate mesh [Fig. 1(a)] modeled by the frequency domain BEM for open surfaces (FD thin plate). Time-step duration and excitation frequency were chosen such that $\Delta_{x}=c \Delta_{t}$ and $\beta=12$.

Agreement between the models is good, though it is recognized that the figure has a broad range of scale and unlike Fig. 7 does not consider phase agreements. Despite the fact that this frequency is slightly below the design frequency of the diffuser $(245 \mathrm{~Hz})$ some grating lobe behavior is visible and predicted by all models. Cancellation of incident and scattered waves at the scattering nulls $\left(\approx \pm 70^{\circ}\right)$ is more complete for the frequency domain results, mirroring the trend inside the body of the uniform welled surface (Fig. 6), suggesting that the frequency domain algorithms are more accurate for time-harmonic problems.

This modeling problem has shown excellent stability and agreement with verified frequency domain BEMs for a geometrically complex real-world acoustic device. In the application of welled surfaces, the surface reflection boundary condition is achieving in the time domain what surface impedance achieves in the frequency domain.

\section{CONCLUSIONS}

The investigation sought to transfer the technique of modeling the well mouths of a Schroeder diffuser as compliant surfaces from the frequency to the time domain. A direct inverse Fourier transform of surface impedance is unsuitable as its convolution kernels are typically noncompact in time and requires future data not available to a time-marching solver. Instead the response of the well to an incoming wave was described by its convolution with a time-invariant kernel denoted surface reflection response, which for a well is extremely compact in time and only requires past data. Pressure and normal velocity at the mouth of the well are readily found from the incoming velocity potential so this was discretized in preference to the more usual choice of total velocity potential. The new surface model was implemented and the algorithm verified on two welled surfaces, one of which was a Schroeder diffuser. Agreement with previously verified frequency domain BEM implementations was good.

It is hoped that with further research this surface model may be generalized to include other classes of compliant obstacle currently modeled as locally reacting surface impedances in the frequency domain. One obstacle is the absence of data for the surface reflection response model when the obstacle is too complex to be considered analytically. Third octave averaged absorption coefficients are usually measured and quoted for most materials, and do not contain enough data to directly reconstruct the surface reflection kernel; instead some form of estimation is required. Drumm and $\mathrm{Lam}^{31}$ and Fung et al. $^{32}$ have both suggested approaches to replacing the convolutions appearing in FDTD surface models with simple filters fitted to the absorption data, and their approaches may also be effective for time domain BEM. Further potential lies in the application of modeling materials with nonlinear and time-variant properties, for which timeharmonic models do not exist.

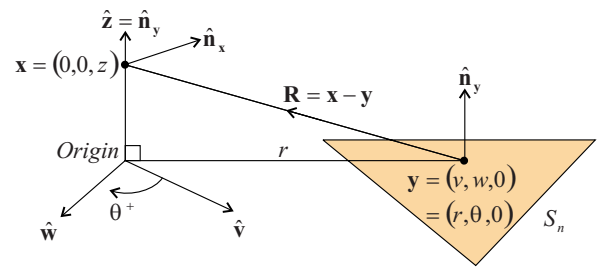

FIG. 9. (Color online) Contour integration geometry and coordinate systems.

\section{ACKNOWLEDGMENTS}

This project was been funded by the UK Engineering and Physical Sciences Research Council (EPSRC) under Grant No. GR/P01144/01.

\section{APPENDIX: NUMERICAL EVALUATION OF INTERACTION COEFFICIENTS}

Accurate evaluation of the interaction coefficients defined in Eq. (21) is fundamental to the accuracy and stability of the algorithm. The temporal basis function chosen has discontinuous derivatives which cause discontinuities and delta functions in the surface integrands, making them unsuitable for solution by Gaussian integration. In addition, the integrand is singular so element self-interaction need often be considered as a special case.

The implementation herein exploits the flat elements and piecewise-constant spatial basis functions to permit regularization of all integrands by coordinate transformation, ${ }^{25,37}$ such that the collocation point is no longer a special case. The radial component of integration is performed analytically, leaving the remaining numerical integration a onedimensional contour integral. This allows an adaptive numerical integration scheme to be used, specifically Simpson integration with Romberg extrapolation. An absolute termination criterion was used, meaning that larger more significant interaction coefficients were evaluated with higher precision than smaller less significant ones. This process is arbitrarily accurate, has better computational cost scaling than two-dimensional integration, and allows the same integration routine to be used for all element pairs as effort is automatically concentrated where necessary.

In order to clarify the conversion of the surface integral over $S_{n}$ into nested integrals two new coordinate systems will be used; one is a Cartesian system $(v, w, z)$ and one a cylindrical polar system $(r, \theta, z)$, both shown in Fig. 9. The origin and positive $z$ direction are the same in both coordinate systems. The origin is defined as the projection of the collocation point $\mathbf{x}$ into the plane of $S_{n}$ and the positive $z$ direction is specified by $\hat{\mathbf{n}}_{\mathbf{y}}$. The positive $v$ direction is defined as the projection of $\hat{\mathbf{n}}_{\mathbf{x}}$ into the plane of $S_{n}$, such that $\hat{\mathbf{w}} \cdot \hat{\mathbf{n}}_{\mathbf{x}}=0$. The positive theta direction is defined such that $v=r \cos (\theta)$ and $w=r \sin (\theta)$ in the conventional way. The variable $z$ refers for the $z$ coordinate of the collocation point $\mathbf{x}$ and any reference to $v, w, r$, or $\theta$ implies the integration point $\mathbf{y}$.

The interaction coefficients are now evaluated according to the following expression, being the sum of integrals over the edges of $S_{n}$ and a contribution from the origin: 


$$
\begin{aligned}
\mathbf{Z}_{l ; m, n}= & \frac{1}{4 \pi} \sum_{\text {edges }} \int_{0}^{1}\left\{\left[\left(1-\alpha-\alpha \hat{\mathbf{n}}_{\mathbf{x}} \cdot \hat{\mathbf{n}}_{\mathbf{y}} \frac{z}{R}\right) \frac{d \theta}{d \mu}+\alpha \hat{\mathbf{n}}_{\mathbf{x}} \cdot \hat{\mathbf{v}} \frac{1}{R} \frac{d w}{d \mu}\right]\left[\left(\frac{z}{R}-1\right) \dot{T}_{j-l}\left(t_{j}-\frac{R}{c}\right)+\left(\frac{z}{R}+1\right) \dot{T}_{j-l}\left(t_{j}-\frac{R+2 d_{n}}{c}\right)\right]\right. \\
& \left.+\alpha \frac{c}{R}\left[\hat{\mathbf{n}}_{\mathbf{x}} \cdot \hat{\mathbf{n}}_{\mathbf{y}}\left(1-\frac{z^{2}}{R^{2}}\right) \frac{d \theta}{d \mu}+\hat{\mathbf{n}}_{\mathbf{x}} \cdot \hat{\mathbf{v}} \frac{z}{R^{2}} \frac{d w}{d \mu}\right]\left[T_{j-l}\left(t_{j}-\frac{R}{c}\right)+T_{j-l}\left(t_{j}-\frac{R+2 d_{n}}{c}\right)\right]\right\} d \mu \\
& -\frac{\theta_{\text {origin }}}{4 \pi}\left[1-\alpha-\alpha \hat{\mathbf{n}}_{\mathbf{x}} \cdot \hat{\mathbf{n}}_{\mathbf{y}} \frac{z}{|z|}\right]\left[\left(\frac{z}{|z|}-1\right) \dot{T}_{j-l}\left(t_{j}-\frac{|z|}{c}\right)+\left(\frac{z}{|z|}+1\right) \dot{T}_{j-l}\left(t_{j}-\frac{|z|+2 d_{n}}{c}\right)\right]
\end{aligned}
$$

Numerical integration is with respect to $\mu$, a dimensionless edge position coefficient varying from zero at the start vertex to one at the end vertex. For an edge e the partial differentials between this and the geometric integration variables are found as follows, where $r_{\perp}$ is the minimum (perpendicular) distance from the origin to the line of edge $\mathbf{e}$ :

$$
\begin{aligned}
& \frac{d \theta}{d \mu}=\frac{|\mathbf{e}| r_{\perp}}{r^{2}} \operatorname{sign}(\hat{\mathbf{e}} \cdot \hat{\boldsymbol{\theta}}), \\
& \frac{d w}{d \mu}=\hat{\mathbf{w}} \cdot \mathbf{e},
\end{aligned}
$$

$\theta_{\text {origin }}$ is the angle the edges of $S_{n}$ make around the origin. This is zero if the origin is outside $S_{n}$ and $2 \pi$ if $S_{n}$ contains the origin. If the origin lies on an edge, $\theta_{\text {origin }}$ will equal the enclosed angle, intersection of one edge implies $\theta_{\text {origin }}=\pi$, and intersection of a corner implies $\theta_{\text {origin }}$ will equal the acute angle between the adjoining edges.

${ }^{1}$ P. D'Antonio and T. J. Cox, "Diffusor application in rooms," Appl. Acoust. 60, 113-142 (2000).

${ }^{2}$ Audio Engineering Society Inc., "AES information document for room acoustics and sound reinforcement systems-Characterisation and measurement of surface scattering uniformity," J. Audio Eng. Soc. AES-4id2001 (r2007).

${ }^{3}$ T. J. Cox and Y. W. Lam, "Prediction and evaluation of the scattering from quadratic residue diffusers," J. Acoust. Soc. Am. 95, 297-305 (1994).

${ }^{4}$ T. J. Cox and P. D'Antonio, Acoustic Absorbers and Diffusers (Spon, London, 2004).

${ }^{5}$ M. B. Friedman and R. P. Shaw, "Diffraction of pulses by cylindrical obstacles of arbitrary cross section," ASME J. Appl. Mech. 29, 40-46 (1962).

${ }^{6} \mathrm{~B}$. P. Rynne, "Instabilities in time marching methods for scattering problems," Electromagnetics 6, 129-144 (1986).

B. P. Rynne and P. D. Smith, "Stability of time marching algorithms for the electric field integral equation," Electromagn. Waves 4, 1181-1205 (1990).

${ }^{8}$ S. J. Dodson, S. P. Walker, and M. J. Bluck, "Implicitness and stability of time domain integral equation scattering analysis," ACES. J. 13, 291-301 (1998).

${ }^{9}$ P. D. Smith, "Instabilities in time marching methods for scattering: cause and rectification," Electromagnetics 10, 439-451 (1990).

${ }^{10}$ H. Wang, D. J. Henwood, P. J. Harris, and R. Chakrabarti, "Concerning the cause of instability in time-stepping boundary element methods applied to the exterior acoustic problem," J. Sound Vib. 305, 289-297 (2007).

${ }^{11}$ A. J. Burton and G. F. Miller, "The application of integral equation methods to the numerical solution of some exterior boundary-value problems," Proc. R. Soc. London, Ser. A 323, 201-210 (1971).

${ }^{12}$ A. A. Ergin, B. Shanker, and E. Michielssen, "Analysis of transient wave scattering from rigid bodies using a Burton-Miller approach," J. Acoust. Soc. Am. 106, 2396-2404 (1999).

${ }^{13}$ A. A. Ergin, B. Shanker, and E. Michielssen, "Fast analysis of transient acoustic wave scattering from rigid bodies using the multilevel plane wave time domain algorithm," J. Acoust. Soc. Am. 107, 1168-1178 (2000).

${ }^{14}$ A. E. Yilmaz, J.-M. Jina, and E. Michielssen, "A fast Fourier transform accelerated marching-on-in-time algorithm for electromagnetic analysis," Electromagnetics 21, 181-197 (2001).

${ }^{15}$ G. C. Herman and P. M. van den Berg, "A least squares iteratively technique for solving time-domain scattering problems," J. Acoust. Soc. Am. 72, 1947-1953 (1982).

${ }^{16}$ Y. Shifman and Y. Leviatan, "On the use of spatio-temporal multiresolution analysis in method of moments solutions of transient electromagnetic scattering," IEEE Trans. Antennas Propag. 49, 1123-1129 (2001).

${ }^{17}$ Y. K. Chung, T. K. Sarkar, B. H. Jung, M. Salazar-Palma, Z. Ji, S. Jang, and K. Kim, "Solution of time domain electric field integral equations using the Laguerre polynomials," IEEE Trans. Antennas Propag. 52, 2319-2328 (2004).

${ }^{18}$ Z. Ji, T. K. Sarkar, B. H. Jung, M. Yuan, and M. Salazar-Palma, "Solving time domain electric field integral equation without the time variable," IEEE Trans. Antennas Propag. 54, 258-262 (2006).

${ }^{19} \mathrm{~S}$. Barmada, "Improving the performance of the boundary element method with time-dependent fundamental solutions by the use of a wavelet expansion in the time domain," Int. J. Numer. Methods Eng. 71, 363-378 (2007).

${ }^{20}$ M. R. Schroeder, "Diffuse sound reflection by maximum-length sequences," J. Acoust. Soc. Am. 57, 149-150 (1975).

${ }^{21}$ M. R. Schroeder, "Binaural dissimilarity and optimum ceilings for concert halls: More lateral sound diffusion," J. Acoust. Soc. Am. 65, 958-963 (1979).

${ }^{22} \mathrm{~A}$. Farina, "A new method for measuring the scattering coefficient and the diffusion coefficient of panels," 86, 928-942 (2000).

${ }^{23}$ J. Redondo, R. Picó, B. Roig, and M. R. Avis, "Time domain simulation of sound diffusers using finite-difference schemes," 93, 611-622 (2007).

${ }^{24}$ R. Martinez, "The thin-shape breakdown (TSB) of the Helmholtz integral equation," J. Acoust. Soc. Am. 90, 2728-2738 (1991).

${ }^{25}$ Y. Kawai and T. Terai, "A numerical method for the calculation of transient acoustic scattering from thin rigid plates," J. Sound Vib. 141, 83-96 (1990).

${ }^{26}$ T. J. Cox and Y. W. Lam, "Prediction and evaluation of the scattering from quadratic residue diffusers," J. Acoust. Soc. Am. 95, 297-305 (1994).

${ }^{27}$ T. Ha-Duong, B. Ludwig, and I. Terrasse, "A Galerkin BEM for transient acoustic scattering by an absorbing obstacle," Int. J. Numer. Methods Eng. 57, 1845-1882 (2003).

${ }^{28} \mathrm{P}$. H. L. Gronenboom, "The applications of boundary elements to steady and unsteady potential fluid flow problems in two and three dimensions," Appl. Math. Model. 6, 35-40 (1982).

${ }^{29}$ R. P. Shaw, "Diffraction of acoustic pulses by obstacles of arbitrary shape with a robin boundary condition," J. Acoust. Soc. Am. 41, 855-859 (1967).

${ }^{30} \mathrm{C}$. K. W. Tam and L. Auriault, "Time-domain impedance boundary conditions for computational aeroacoustics," AIAA J. 34, 917-923 (1996).

${ }^{31}$ I. A. Drumm and Y. W. Lam, "Development and assessment of a finite difference time domain room acoustic prediction model that uses hall data in popular formats," Proceedings of Internoise, Turkey, 2007.

${ }^{32}$ K.-Y. Fung, H. Ju, and B.-P. Tallapragada, "Impedance and its timedomain extensions," AIAA J. 38, 30-38 (2000).

${ }^{33}$ A. D. Pierce, Acoustics: An Introduction to Its Physical Principles and Application (McGraw-Hill, London, 1981).

${ }^{34}$ D. A. Vechinski and S. M. Rao, "Transient scattering from dielectric cylinders: E-field, h-field and combined-field solutions," Radio Sci. 27, 611622 (1992). 
${ }^{35}$ D. J. Chappell, P. J. Harris, D. Henwood, and R. Chakrabarti, “A stable boundary integral equation method for modeling transient acoustic radiation," J. Acoust. Soc. Am. 120, 74-80 (2006).

${ }^{36}$ T. J. Cox and Y. W. Lam, "Evaluation of methods for predicting the scat- tering from simple rigid panels," Appl. Acoust. 40, 123-140 (1993).

${ }^{37}$ Y. Ding, A. Forestier, and T. Ha-Duong, "A Galerkin scheme for the time domain integral equation of acoustic scattering from a hard surface," J. Acoust. Soc. Am. 86, 1566-1572 (1989). 